\section{Diabetes and periodontal disease: a two-way relationship}

\author{
L. Casanova, ${ }^{1}$ F. J. Hughes ${ }^{1}$ and P. M. Preshaw ${ }^{* 2}$
}
IN BRIEF
- Explains the current knowledge regarding the links between diabetes and periodontal disease.
- Provides information on the main types of diabetes and explains how to interpret blood glucose and glycated haemoglobin values that patients may report to you.
- Gives practical guidance for the dental team in assessing and managing periodontal status in people with diabetes.

Periodontitis and diabetes are common, complex, chronic diseases with an established bidirectional relationship. That is, diabetes (particularly if glycaemic control is poor) is associated with an increased prevalence and severity of periodontitis, and, severe periodontitis is associated with compromised glycaemic control. Periodontal treatment (conventional nonsurgical periodontal therapy) has been associated with improvements in glycaemic control in diabetic patients, with reductions in $\mathrm{HbA} 1 \mathrm{c}$ of approximately $0.4 \%$ following periodontal therapy. For these reasons, management of periodontitis in people with diabetes is particularly important. The dental team therefore has an important role to play in the management of people with diabetes. An emerging role for dental professionals is envisaged, in which diabetes screening tools could be used to identify patients at high risk of diabetes, to enable them to seek further investigation and assessment from medical healthcare providers.

\section{INTRODUCTION}

Periodontitis and diabetes are both highly prevalent conditions, and the association between these two common diseases has been recognised by dental professionals for many years. Epidemiological studies have clearly identified that diabetes is a major risk factor for periodontitis, increasing the risk approximately three-fold compared to non-diabetic individuals, particularly if glycaemic control is poor. ${ }^{1}$ In recent years, the precise relationship between periodontitis and diabetes has been the subject of much interest, given that both conditions are highly prevalent, and also because it has become increasingly clear that there are interactions between the two diseases that have important clinical implications for dental professionals, physicians and, most importantly, patients. This narrative review aims to summarise our current understanding of the relationship between diabetes and periodontitis and to discuss the clinical implications of these findings for the dental professional. Relevant literature was identified from Medline and PubMed database

'King's College London, Floor 21 Tower Wing, Guy's Hospital, Great Maze Pond, London SE1 9RT; ${ }^{2 *}$ School of Dental Sciences, Newcastle University, Framlington Place, Newcastle upon Tyne, NE2 4BW

*Correspondence to: Philip Preshaw

Email: philip.preshaw@newcastle.ac.uk

\section{Refereed Paper}

Accepted 22 July 2014

DOI: 10.1038/sj.bdj.2014.907

${ }^{\circledR}$ British Dental Journal 2014; 217: 433-437 searches together with scrutiny of reference lists from published articles.

\section{PERIODONTAL DISEASE}

Inflammatory periodontal diseases are the most common chronic inflammatory conditions of man, affecting - if including gingivitis as well as periodontitis - up to $90 \%$ of the world's population. ${ }^{2}$ When considering severe periodontitis (which typically refers to the presence of pocketing $\geq 6 \mathrm{~mm}$ ), the prevalence is generally estimated to be around 5-15\% of adults globally. ${ }^{3}$ Consistent with this are the findings of the 2009 Adult Dental Health Survey for England, Wales and Northern Ireland, which identified that $8 \%$ of adults have at least one pocket of $6 \mathrm{~mm}$ or deeper. ${ }^{4}$ The inflammation in the periodontal tissues that characterises periodontitis is initiated by the accumulation of the subgingival biofilm; however, susceptibility to disease is determined by a number of factors independent of the absolute levels of plaque. Pre-eminent among these are the major environmental risk factors for periodontitis, tobacco smoking ${ }^{5}$ and diabetes. ${ }^{6}$

The tissue damage that results from the chronic inflammation in the periodontal tissues (loss of attachment, breakdown of periodontal ligament fibres and alveolar bone resorption) is largely irreversible. It is also typically painless, so may remain unnoticed for many years unless the patient is seen by a dental healthcare professional. The consequences of periodontitis, such as gingival bleeding, compromised aesthetics, recurrent periodontal infections, tooth mobility and tooth loss, may all have negative impacts on daily living and quality of life, with implications for function, comfort, self-confidence, social interactions and food choices. ${ }^{7-9}$

\section{DIABETES}

Diabetes is a group of metabolic disorders characterised by hyperglycaemia (elevated blood sugar). The main types of diabetes are type 1 diabetes, type 2 diabetes and gestational diabetes.

Type 1 diabetes (in the past, referred to as insulin-dependent diabetes, or juvenile diabetes) describes a condition in which there is a failure to produce insulin as a result of autoimmune destruction of the insulinproducing $\beta$-cells in the pancreas. Genetic susceptibility is a major risk factor in type 1 diabetes, and in susceptible individuals, the onset of diabetes appears to be triggered by environmental factors such as viral infections and diet, rather than being related to lifestyle factors. The onset of type 1 diabetes is usually in childhood or young adulthood. Type 1 diabetes constitutes about 5-10\% of all cases of diabetes, but accounts for more than $90 \%$ of diabetes cases in young people less than 25 years old. Complications arise as a result of hyperglycaemia and include acute conditions such as diabetic ketoacidosis, as well as chronic disorders such as nephropathy, neuropathy, cardiovascular disease, and acute coronary syndrome. Many patients with type 1 diabetes do not develop serious long-term complications, however, 
particularly if their blood glucose levels are well controlled. The condition is typically managed by blood glucose monitoring and insulin therapy. ${ }^{10}$

Type 2 diabetes (previously referred to as non-insulin-dependent diabetes, or adult onset diabetes) results from insulin resistance; that is, there is reduced responsiveness of the cells in the body to insulin, leading to a reduced capacity to transfer glucose out of the circulation and into cells. This leads to hyperglycaemia (elevated blood glucose levels). In the early stages, insulin secretion by the $\beta$-cells of the pancreas may be normal, but this can diminish over time, leading to insulin deficiency as well as insulin resistance. Type 2 diabetes constitutes 90-95\% of all diabetes cases, and is typically associated with lifestyle factors such as overweight/ obesity and lack of exercise, as well as genetic factors. The management of type 2 diabetes typically involves combinations of lifestyle change, weight loss, dietary modification, oral hypoglycaemic drugs and, in severe cases, insulin injections. The age of onset of type 2 diabetes was previously typically considered to be in the 40s and 50s, but increasing numbers of cases in younger age groups are now being identified.

Gestational diabetes is a form of diabetes that occurs in pregnant women without a previous history of diabetes who develop hyperglycaemia during their pregnancy. It is characterised by reduced insulin secretion as well as insulin resistance, and usually improves after pregnancy, though a small proportion of affected women may be found to have diabetes (usually type 2) after their pregnancy.

The adverse effects of diabetes are associated with the hyperglycaemia that characterises the condition. Diabetes has negative impacts on multiple body systems and disease states throughout the body, including cardiovascular disease, renal disease, peripheral vascular disease, ocular disease and neuropathy. The level of glycaemic control is routinely assessed by measuring glycated haemoglobin (HbA1c) in the blood. This has traditionally been expressed as a percentage, being the percentage of haemoglobin that has glucose molecules absorbed onto the haemoglobin molecule, that is, the percentage of haemoglobin that is 'glycated'. However, the way that HbA1c values are reported has now switched from a percentage to a measurement in mmols/ mol (Table 1). The lifespan of a red blood cell is typically around three months, and therefore HbA1c measurements give an indication of the level of glycaemic control over that period. In a non-diabetic person, HbA1c is typically around 5.5\% $(37 \mathrm{mmol} /$ mol). In people with diabetes, HbA1c levels of $<7.0 \%$ (53 mmol $/ \mathrm{mol}$ ) would typically indicate good glycaemic control (though many clinicians will strive to work with their patients to achieve $\mathrm{HbA} 1 \mathrm{c}<6.5 \%, 48 \mathrm{mmol} /$ mol). Levels of $8-9 \%(64-75 \mathrm{mmol} / \mathrm{mol})$ or higher indicate poor glycaemic control. The complications of diabetes are closely linked to the level of glycaemic control, and it has been shown that each $1 \%$ reduction in $\mathrm{HbA1c}$ has been associated with measurable reductions in risk of diabetes complications, a $21 \%$ reduction in deaths related to diabetes, a 14\% reduction for myocardial infarction, and a 37\% reduction for microvascular complications of diabetes. ${ }^{11}$ It is, therefore, extremely important to work with patients to optimise their glycaemic control.

We are currently witnessing a global epidemic of type 2 diabetes, with huge increases in the numbers of people affected in countries throughout the world. This has major implications for provision of healthcare services, as well as individual impacts in terms of life expectancy, morbidity, quality of life and healthcare costs. It is estimated that currently 347 million people suffer from diabetes worldwide ${ }^{12}$ and this figure is predicted to rise to approximately 439 million, almost $10 \%$ of adults, by 2030 . In the UK it is estimated that about $6.5 \%$ of the total population are affected by diabetes. ${ }^{13}$

\section{THE EFFECTS OF DIABETES ON PERIODONTAL DISEASE}

Epidemiological studies have consistently shown that diabetes is associated with increased risk of periodontitis. The majority of research has focused on type 2 diabetes, although type 1 diabetes appears to have an identical effect on risk for periodontitis. The magnitude of the increased risk of periodontitis is known to be dependent on the level of glycaemic control, as it is with the risk of all complications of diabetes. Thus, in well controlled diabetes with HbA1c of around $7 \%$ (53 mmol $/ \mathrm{mol}$ ) or lower, there appears to be little effect of diabetes on risk for periodontitis. However, the risk increases exponentially as glycaemic control deteriorates. Overall, the increased risk of periodontitis in patients with diabetes is estimated to be between 2-3 fold - that is, it increases the risk for periodontitis by 2-3 times. ${ }^{1,14}$

Diabetes increases the prevalence of periodontitis, the extent of periodontitis (that is, number of affected teeth) and the severity of the disease. It has been reported that patients with diabetes may present to the dental professional with multiple recurring periodontal abscesses, and although this may sometimes be the case, it is not typical. Thus, there is not normally any particular
Table 1 Measurement of blood glucose: what do the numbers mean?

Glycated haemoglobin ( $\mathrm{HbA} 1 \mathrm{c})$

Measuring $\mathrm{HbA} 1 \mathrm{c}$ indicates how much haemoglobin in the blood has become glycated (chemically bonded with glucose)

It provides an indication of blood glucose levels over the last three months (this being the life span of a red blood cell)

Traditionally, this was expressed as a percentage (the \% of haemoglobin that was glycated), but there has now been a switch to express $\mathrm{HbA} 1 \mathrm{c}$ in $\mathrm{mmol} / \mathrm{mol}$

Example $\mathrm{HbA} 1 \mathrm{c}$ values expressed as a percentage and as $\mathrm{mmol} / \mathrm{mol}$ :

$5 \%=31 \mathrm{mmol} / \mathrm{mol}$

$6 \%=42 \mathrm{mmol} / \mathrm{mol}$

$7 \%=53 \mathrm{mmol} / \mathrm{mol}$

$8 \%=64 \mathrm{mmol} / \mathrm{mol}$

$9 \%=75 \mathrm{mmol} / \mathrm{mol}$

Blood glucose measurements

Blood glucose is measured on a regular basis by people with diabetes as part of their daily self-monitoring

It measures the concentration of glucose in the blood at the time of the test, and is measured in $\mathrm{mmol} / \mathrm{l}$ (millimoles per litre)

The aim is to achieve blood glucose concentrations that are as close as possible to non-diabetic values

Target values are approximately 4-7 $\mathrm{mmol} / \mathrm{l}$ before meals, and below $8.0-8.5 \mathrm{mmol} / \mathrm{l}$ two hours after meals. Target levels are individual to each person, and will be agreed between the person and their diabetes care team.

characteristic clinical presentation of periodontitis in patients with diabetes other than the normal clinical features of the condition.

In addition to the effects of diabetes on periodontitis, various other oral conditions may also be associated with diabetes. Many patients with diabetes may also take calcium channel blocker drugs such as amlodipine and nifedipine for hypertension, and this may result in gingival overgrowth in some cases. Occasionally, medications can also have other oral manifestations, such as lichenoid mucosal reactions to metformin. Other oral consequences of diabetes may include xerostomia resulting in increased risk for caries, candidal infections and chronic mouth ulcers.

The mechanisms that link diabetes and periodontitis are not completely understood, but involve aspects of inflammation, immune functioning, neutrophil activity, and cytokine biology. ${ }^{15}$ Both type 1 and type 2 diabetes are associated with elevated levels of systemic markers of inflammation. ${ }^{16}$ Diabetes increases inflammation in periodontal tissues, with higher levels of inflammatory mediators such as interleukin- $1 \beta$ (IL-1 $1 \beta$ ) and tumour necrosis factor- $\alpha$ (TNFa). ${ }^{17},{ }^{18}$ Periodontal disease has been associated with higher levels of inflammatory 
mediators such as TNF- $\alpha$ in people with diabetes. ${ }^{19}$ Accumulation of reactive oxygen species, oxidative stress, and interactions between advanced glycation end products (AGEs) in the periodontal tissues and their receptor (RAGE, the receptor for advanced glycation end products) all contribute to increased inflammation in the periodontal tissues in people with diabetes. A detailed review of the pathogenic mechanisms that link diabetes and periodontitis is beyond the scope of this article but this subject area has been recently reviewed. ${ }^{15}$

\section{THE EFFECTS OF PERIODONTAL DISEASE ON DIABETES}

Evidence to support a negative impact of periodontal disease on diabetes was first postulated following studies of the Gila River Indian Community, a population of Native Americans with a high prevalence of diabetes. It was noted that severe periodontitis was associated with increased risk of poor glycaemic control (HbA1c >9.0\%, $75 \mathrm{mmol} /$ mol) at follow-up (minimum of two years later), suggesting that periodontitis may be compromising diabetes control..$^{20}$ Other studies have reported increased prevalence of diabetes complications, such as cardiovascular complications, retinopathy, neuropathy and proteinuria in people with advanced periodontitis. ${ }^{21-24}$ More recent studies of people with type 2 diabetes from the Gila River Indian Community identified that the incidences of macroalbuminuria were 2.0, 2.1 and 2.6 times as high in those who also had moderate periodontitis, severe periodontitis, or who were edentulous, respectively, compared to those with no/mild periodontitis $(\mathrm{p}<0.05)$. Furthermore, the incidences of endstage renal disease (ESRD) were 2.3, 3.5 and 4.9 times as high for those with moderate or severe periodontitis, or who were edentulous, respectively $(\mathrm{p}<0.05)$. The authors concluded that moderate and severe periodontitis and edentulousness predicted the occurrence of nephropathy (characterised by macroalbuminuria and ESRD) in a 'dose-dependent' manner in the individuals with type 2 diabetes. ${ }^{25}$

The impact of periodontitis on deaths from cardiovascular complications and diabetic nephropathy has also been investigated in a longitudinal study of Pima Indians with type 2 diabetes. Age- and sex-adjusted death rates (deaths per 1,000 person-years) were 3.7 for those with no/mild periodontitis, 19.6 for those with moderate periodontitis and 28.4 for those with severe periodontitis. After adjustment for known confounders, it was shown that diabetic individuals with severe periodontitis had 3.2 times increased risk of cardiorenal mortality (ischaemic heart disease and diabetic nephropathy combined) compared with the reference group (those with no periodontitis, mild and moderate periodontitis combined). ${ }^{26}$

Changes in HbA1c in non-diabetic individuals who were monitored for a period of five years have also been associated with the presence of periodontitis. In a longitudinal study, the (non-diabetic) participants with the most advanced periodontitis at baseline were found to have a five times greater increase in their $\mathrm{HbA1c}$ values over five years (change in $\mathrm{HbA} 1 \mathrm{c} 0.106 \pm 0.03 \%$ ) compared to those who did not have periodontitis at baseline (change in HbA1c $0.023 \pm 0.02 \%) .{ }^{27}$ This is the first study to suggest that periodontitis results in increased HbA1c levels in individuals who do not have diabetes, and is continuing further to identify whether this translates into an increased occurrence of incident diabetes (new cases of diabetes).

\section{THE EFFECT OF PERIODONTAL TREATMENT ON DIABETES CONTROL}

A large number of studies have now been carried out to investigate the effects of treating periodontitis on glycaemic control in people with diabetes. Some of these have been performed as randomised controlled trials, in which periodontal treatment was compared to no periodontal treatment (or delayed periodontal treatment) in people with diabetes and periodontitis. To date, up to seven systematic reviews and meta-analyses have been published which investigated in detail the outcomes of these studies, and a consistent finding has been that periodontal treatment is associated with reductions in $\mathrm{HbA} 1 \mathrm{c}$ of the order of $0.4 \% \cdot{ }^{28-34}$ One of these studies was a Cochrane review, which similarly identified a reduction in HbA1c of approximately $0.4 \%$ following non-surgical periodontal therapy. ${ }^{33}$ Although such an improvement in HbA1c may appear to be relatively modest, it may, in fact, have very significant clinical impacts, because, as reported above, every $1 \%$ reduction in HbA1c is associated with a measurably reduced risk for diabetes complications. ${ }^{11}$ Furthermore, periodontal treatment is a relatively straightforward clinical intervention, that doesn't have unwanted effects that might be associated with additional medications taken as part of diabetes therapy.

However, it is recognised that not all clinical trials which assessed the impact of periodontal therapy on glycaemic control have identified similar findings, and in particular, a recent multi-centre study of over 500 patients failed to demonstrate any benefit of periodontal treatment on glycaemic control. ${ }^{35}$ However, this study has been criticised on account of three main issues: (i) for recruiting patients with moderately good glycaemic control already (HbA1c <9\%, $75 \mathrm{mmol} / \mathrm{mol}$ ) who would therefore have limited potential for improvement following periodontal treatment; (ii) for achieving only a relatively poor response to the periodontal treatment; and (iii) for having a very overweight/obese study population (average body mass index [BMI] of approximately $35 \mathrm{~kg} / \mathrm{m}^{2}$ in the treatment group, indicating marked obesity, which would mask any decrease in inflammatory response resulting from periodontal treatment). ${ }^{36}$ It is clear that further evidence is required to address the specific question of the impact of periodontal treatment on glycaemic control.

\section{IMPLICATIONS FOR THE DENTAL PROFESSIONAL}

In 2007, the World Health Organisation (WHO) Executive Board acknowledged the intrinsic link between oral health, general health and quality of life. ${ }^{37}$ It has been suggested that oral health is a neglected area of global health, and an editorial in The Lancet proposed that promoting and improving oral health should be part of the routine agenda of healthcare policymakers and clinicians. ${ }^{38}$ Periodontal disease and diabetes are directly and independently associated chronic diseases of high prevalence in the population, and the global prevalence of type 2 diabetes, in particular, is rising dramatically. In 2000, the US Surgeon General referred to a 'silent epidemic' of oral and dental diseases, and stressed the importance of oral health as being essential for general health and well-being. ${ }^{39}$

A patient with diabetes may have a number of specific direct implications for the dental professional:

- Patients with (particularly

type 1) diabetes may be at risk of hypoglycaemic episodes while attending the dental surgery

- People with diabetes are at higher risk of oral disease, particularly periodontitis, and particularly if their diabetes is poorly controlled

- Patients with undiagnosed diabetes may present at the dental surgery and provide an opportunity for referral for opportunistic screening based on the presence of periodontal disease and other diabetic risk factors

- Patients with diabetes may experience some improvement in their glycaemic control following successful periodontal treatment.

The management of medical emergencies involving diabetic patients in the dental 
practice setting, particularly hypoglycaemic episodes, has been addressed in detail ${ }^{40,41}$ and will not be repeated in this paper.

Regarding increased susceptibility to periodontitis in people with diabetes, this has a number of implications for dental management. Firstly, it is important that diabetic patients are aware of the potential impact their condition may have on their oral and periodontal health. Patients who are newly diagnosed with diabetes should be told about this impact and we should continue to urge our medical colleagues to recommend a dental examination to their patients. Unfortunately, many patients with diabetes and also many medical clinicians are unaware of the links between periodontitis and diabetes, and of the potential benefits that periodontal treatment may have for diabetic patients. ${ }^{42,43}$

Routine periodontal assessment should be performed in all patients, including those with diabetes. Ask the patient about their level of glycaemic control: many will be able to tell you their most recent HbA1c measurements. If the patient does not have periodontitis, then long term preventive care and monitoring should be undertaken (that is, same as for all patients). Diabetic patients should also be evaluated for the other potential oral complications of diabetes, including caries, dry mouth, burning mouth, candidal infections and co-morbidities such as those associated with medications.

If periodontitis is diagnosed, it should be managed as appropriate. This would typically involve (similar to non-diabetic patients) patient education and empowerment, oral hygiene instruction, non-surgical therapy (root surface debridement), and monitoring of treatment outcomes. Effective periodontal treatment is particularly important in people with diabetes, given that periodontitis has potential negative impacts on glycaemic control and diabetes complications, and that periodontal treatment has been associated with improvements in HbA1c.

In most cases, conventional (non-surgical) periodontal treatment is very effective in diabetic patients, including optimisation of plaque control to control the inflammation that leads to periodontal destruction along with a major emphasis on self-management and patient education. There is little or no evidence to suggest, for example, that diabetic patients require antibiotics as part of periodontal therapy.

The escalating human and economic burden of diabetes requires a multidisciplinary approach for the prevention, diagnosis and management of the disease and its complications including periodontitis. Many cases of diabetes in the UK are undiagnosed and therefore, the dental health professional may have a useful role to play in opportunistic screening of dental patients for risk of diabetes. A study from the USA has demonstrated that a combination of age (over 45), presence of periodontal disease and at least one other diabetic risk factor (for example, positive family history, self-reported BMI $>25$, hypertension) was very successful in identifying undiagnosed cases of diabetes. ${ }^{44}$

Recently, a diabetes screening programme was undertaken in dental patients in different clinical settings in the UK (general dental practices, a dental hospital clinic, and a dental school outreach clinic). ${ }^{45}$ The dental clinicians performed the screening procedure, and patients who were determined to be at moderate or high risk of diabetes were recommended to visit their medical GP for further investigation. This study identified that dental professionals who were trained in the screening procedure valued this process, as did the patients, who received the diabetes screening in the dental setting very favourably. The major downside was the time required to perform the screening, which added around 20 minutes to the length of each dental appointment. Clearly that would not be practical within current contractual arrangements for NHS dental practices, but it does highlight that dental healthcare teams could potentially play an important role in screening their dental patients for systemic conditions such as diabetes.

\section{CONCLUSIONS}

Diabetes increases the risk for periodontitis (particularly if poorly controlled) and evidence suggests that advanced periodontitis also compromises glycaemic control. Periodontal treatment has been associated with improvements in glycaemic control (with HbA1c reductions of approximately $0.4 \%$ reported in systematic reviews and meta-analyses), though more research is required to investigate this further. Oral health (including periodontal health) is a fundamentally important component of general health, and particularly so in diabetes. Periodontal assessment is as important in people with diabetes as it is in people who do not have diabetes, and people with diabetes should be made aware of their increased risk for periodontal disease. The dental team has an important role to play in the management of people with diabetes. An emerging role for the dental team is envisaged in which, through the use of relatively simple screening tools, they may help to identify patients at high risk of diabetes.

1. Mealey B L, Ocampo G L. Diabetes mellitus and periodontal disease. Periodontol 20002007 ; 44: 127-153.

2. Pihlstrom B L, Michalowicz B S, Johnson N W. Periodontal diseases. Lancet 2005; 366: 1809-1820.
3. Dye B A. Global periodontal disease epidemiology Periodontol 2000 2012; 58: 10-25.

4. White D, Pitts N, Steele J G et al. Diseases and related disorders - a report from the Adult Dental Health Survey 2009. London: NHS Information Centre for Health and Social Care, 2011.

5. Warnakulasuriya S, Dietrich $\mathrm{T}$, Bornstein M M et al. Oral health risks of tobacco use and effects of cessation. Int Dent J 2010; 60: 7-30.

6. Preshaw P M, Alba A L, Herrera D et al. Periodontitis and diabetes: a two-way relationship. Diabetologia 2012; 55: 21-31.

7. Jowett A K, Orr M T, Rawlinson A, Robinson P G. Psychosocial impact of periodontal disease and its treatment with $24 \mathrm{~h}$ root surface debridement. J Clin Periodontol 2009; 36: 413-418.

8. Needleman I, McGrath C, Floyd P, Biddle A. Impact of oral health on the life quality of periodontal patients. J Clin Periodontol 2004; 31: 454-457.

9. O'Dowd L K, Durham J, McCracken G I, Preshaw P M Patients' experiences of the impact of periodontal disease. J Clin Periodonto/ 2010; 37: 334-339.

10. NICE. Type 1 diabetes: Diagnosis and management of type 1 diabetes in children, young people and adults. NICE guidelines (CG15). July 2004. Available at: http://www.nice.org.uk/guidance/CG15 (accessed 16 July 2014).

11. Stratton I M, Adler A I, Neil H A et al. Association of glycaemia with macrovascular and microvascular complications of type 2 diabetes (UKPDS 35): prospective observational study. BMJ 2000; 321: 405-412.

12. WHO. World Health Organisation. Diabetes. Fact sheet No. 312. Reviewed October 2013. Available at: http://www.who.int/mediacentre/factsheets/fs312/ en/ (accessed 16 July 2014).

13. IDF. Internation Diabetes Federation. IDF diabetes atlas, 6th ed. 2013. http://www.idf.org/sites/default/ files/DA6_Regional_factsheets_0.pdf (accessed 16 July 2014).

14. Tsai C, Hayes C, Taylor G W. Glycaemic control of type 2 diabetes and severe periodontal disease in the US adult population. Community Dent Oral Epidemio/ 2002; 30: 182-192.

15. Taylor J J, Preshaw P M, Lalla E. A review of the evidence for pathogenic mechanisms that may link periodontitis and diabetes. J Clin Periodonto/ 2013; 40 (Suppl 14): S113-134.

16. Dandona P, Aljada A, Bandyopadhyay A. Inflammation: the link between insulin resistance, obesity and diabetes. Trends Immuno/ 2004; 25: 4-7.

17. Engebretson S P, Hey-Hadavi J, Ehrhardt F J et al. Gingival crevicular fluid levels of interleukin $1 \beta$ and glycaemic control in patients with chronic periodontitis and type 2 diabetes. J Periodontol 2004; 75: 1203-1208.

18. Salvi G E, Collins J G, Yalda B et al. Monocytic TNFa secretion patterns in IDDM patients with periodontal diseases. J Clin Periodontol 1997; 24: 8-16.

19. Engebretson S, Chertog R, Nichols A, Hey-Hadavi J, Celenti $R$, Grbic J. Plasma levels of tumour necrosis factor-alpha in patients with chronic periodontitis and type 2 diabetes. J Clin Periodonto/ 2007; 34: 18-24.

20. Taylor G W, Burt B A, Becker M P et al. Severe periodontitis and risk for poor glycaemic control in patients with non-insulin-dependent diabetes mellitus. J Periodonto/ 1996; 67: 1085-1093.

21. Karjalainen $\mathrm{KM}$ M, Knuuttila M L, von Dickhoff $\mathrm{K} \mathrm{J}$. Association of the severity of periodontal disease with organ complications in type 1 diabetic patients. J Periodontol 1994; 65: 1067-1072.

22. Moore P A, Weyant R J, Mongelluzzo M B et al. Type 1 diabetes mellitus and oral health: assessment of tooth loss and edentulism. J Public Health Dent 1998; 58: 135-142.

23. Moore P A, Weyant R J, Mongelluzzo M B et al. Type 1 diabetes mellitus and oral health: assessment of periodontal disease. J Periodontol 1999; 70: 409-417.

24. Thorstensson $H_{1}$ Kuylenstierna J, Hugoson A. Medical status and complications in relation to periodontal disease experience in insulin-dependent diabetics. J Clin Periodontol 1996; 23: 194-202. 
25. Shultis W A, Weil E J, Looker H C et al. Effect of periodontitis on overt nephropathy and end-stage renal disease in type 2 diabetes. Diabetes Care 2007; 30: 306-311.

26. Saremi A, Nelson R G, Tulloch-Reid M et al. Periodontal disease and mortality in type 2 diabetes. Diabetes Care 2005; 28: 27-32.

27. Demmer R T, Desvarieux M, Holtfreter B et al. Periodontal status and $A 1 C$ change: Iongitudinal results from the study of health in Pomerania (SHIP). Diabetes Care 2010; 33: 1037-1043.

28. Darre L, Vergnes J N, Gourdy P, Sixou M. Efficacy of periodontal treatment on glycaemic control in diabetic patients: A meta-analysis of interventional studies. Diabetes Metab 2008; 34: 497-506.

29. Engebretson S, Kocher T. Evidence that periodontal treatment improves diabetes outcomes: a systematic review and meta-analysis. J Clin Periodonto/ 2013; 40 Suppl 14: S153-163.

30. Janket S J, Wightman A, Baird A E, Van Dyke T E, Jones J A. Does periodontal treatment improve glycaemic control in diabetic patients? A metaanalysis of intervention studies. J Dent Res 2005; 84: 1154-1159.

31. Liew A K, Punnanithinont N, Lee Y C, Yang J. Effect of non-surgical periodontal treatment on HbA1c: a meta-analysis of randomized controlled trials. Aust Dent J 2013; 58: 350-357.
32. Sgolastra F, Severino M, Pietropaoli D, Gatto R, Monaco A. Effectiveness of periodontal treatment to improve metabolic control in patients with chronic periodontitis and type 2 diabetes: a meta-analysis of randomized clinical trials. J Periodonto/ 2013; 84: 958-973.

33. Simpson T C, Needleman I, Wild S H, Moles D R, Mills E J. Treatment of periodontal disease for glycaemic control in people with diabetes. Cochrane Database Syst Rev 2010; CD004714.

34. Teeuw W J, Gerdes V E A, Loos B G. Effect of periodontal treatment on glycaemic control of diabetic patients: a systematic review and meta-analysis. Diabetes Care 2010; 33: 421-427.

35. Engebretson S P, Hyman L G, Michalowicz B S et al. The effect of nonsurgical periodontal therapy on haemoglobin A1c levels in persons with type 2 diabetes and chronic periodontitis: a randomized clinical trial. JAMA 2013; 310: 2523-2532.

36. Borgnakke W S, Chapple I L C, Genco R J et al. The multi-center randomized controlled trial (RCT) published by the Journal of the American Medical Association (JAMA) on the effect of periodontal therapy on glycated hemoglobin ( $\mathrm{HbA} 1 \mathrm{C}$ ) has fundamental problems. J Evid Based Dent Pract 2014; 14: 127-132.

37. World Health Organisation. Oral health: action plan for promotion and integrated disease prevention (EB120/10). 120th Session, 22-30 January. Geneva:
WHO, 2007.

38. Oral health: prevention is key. Lancet 2009; 373: 1.

39. US Department of Health and Human Services. Oral health in America: a report of the surgeon general. Rockville: US Department of Health and Human Services, National Institute of Dental and Craniofacial Research, National Institutes of Health, 2000.

40. Greenwood M. Medical emergencies in the dental practice. Periodontol 2000 2008; 46: 27-41.

41. Greenwood M. Medical emergencies in dental practice: 2. Management of specific medical emergencies. Dent Update 2009; 36: 262-264, 266-268.

42. Allen E M, Ziada HM, O'Halloran D, Clerehugh V Allen P F. Attitudes, awareness and oral healthrelated quality of life in patients with diabetes. J Oral Rehabil 2008; 35: 218-223.

43. Bissett S M, Stone K M, Rapley T, Preshaw P M. An exploratory qualitative interview study about collaboration between medicine and dentistry in relation to diabetes management. BMJ Open 2013; 3: e002192.

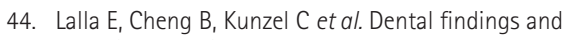
identification of undiagnosed hyperglycaemia. J Dent Res 2013; 92: 888-892.

45. Wright $D$, Muirhead V, Weston-Price S, Fortune F. Type 2 diabetes risk screening in dental practice settings: a pilot study. Br Dent J 2014; 216: E15. 